\title{
e-Phaïstos
}

e-Phaïstos

Revue d'histoire des techniques / Journal of the history

of technology

IX-2 | 2021

Quel objet pour quel musée?

\section{La guerre du froid a bien eu lieu...}

Retour sur un pan de l'histoire technique et culturelle du réfrigérateur The War of Cold has Indeed Taken Place... A Part of the Technical and Cultural History of the Refrigerator

\section{Aurélie Brayet}

\section{(2) OpenEdition}

\section{Journals}

Édition électronique

URL : https://journals.openedition.org/ephaistos/9183

DOI : 10.4000/ephaistos.9183

ISSN : 2552-0741

Éditeur

IHMC - Institut d'histoire moderne et contemporaine (UMR 8066)

Référence électronique

Aurélie Brayet, "La guerre du froid a bien eu lieu... », e-Phaïstos [En ligne], IX-2 | 2021, mis en ligne le 26 octobre 2021, consulté le 28 octobre 2021. URL : http://journals.openedition.org/ephaistos/9183 ; DOI : https://doi.org/10.4000/ephaistos. 9183

Ce document a été généré automatiquement le 28 octobre 2021.

Tous droits réservés 


\title{
La guerre du froid a bien eu lieu...
}

\author{
Retour sur un pan de l'histoire technique et culturelle du réfrigérateur \\ The War of Cold has Indeed Taken Place... A Part of the Technical and Cultural \\ History of the Refrigerator
}

Aurélie Brayet

1 Les objets de la vie domestique et du quotidien et particulièrement ceux issus du monde ménager, ont été négligés par l'histoire des techniques, n'occupant généralement que quelques pages où ils sont convoqués à titre d'illustration. Pourtant, frigos, cocotteminute, plaques à induction et autres micro-ondes ont été bien souvent le principal contact de tout à chacun avec la technologie. Ce sont des objets techniques omniprésents dans la maison et particulièrement en cuisine. Le réfrigérateur est un de ces objets invisibles. Invisibles, parce que désormais «intégrés ", cachés derrière les placages des cuisines, et parce que rarement sujet d'étude de recherche ${ }^{1}$. Les historiens Roger Thévenot dans les années 1970 (Thévenot 1978), Ruth Schwartz Cowan (Schwartz Cowan 1985) dans les années 1980 et la sociologue Quynh Delaunay (Delaunay 2003) au début des années 2000 ont apporté des éclairages essentiels sur cet humble "frigo", que nous ne voyons plus. Ils ont imposé le réfrigérateur comme un objet technique, culturel et social complexe, digne d'être étudié (Brayet 2019).

Néanmoins, au-delà d'être le récit d'une aventure technique, sociale et culturelle qui traverse les époques et raconte celle des familles, des nations et finalement la "grande histoire ", l'histoire du réfrigérateur est aussi la chronique de " guerres » scientifiques et techniques. Au cours du XIX siècle, un véritable besoin en froid imposé par le développement de secteurs industriels nouveaux comme le transport frigorifique de viande sur les océans, le développement des wagons frigorifiques sur rail (Carrière 2010) ou encore l'essor des brasseries ${ }^{2}$, de nouveaux comportements (consommation, conservation, hygiénisme, peur du microbe, etc.) et le déploiement d'une mondialisation de l'approvisionnement alimentaire, poussent les États dans une course à la conquête de nouvelles sources d'approvisionnement en glace naturelle et orientent la recherche scientifique et technique vers le domaine du froid. Durant la majeure partie du XIX' siècle, une guerre ${ }^{3}$ (Thévenot 1978, Blain 2006) oppose chercheurs et industriels du monde entier dans la quête de solutions pour améliorer la production 
artificielle de froid, l'industrialiser et ainsi la diffuser largement à tous. Le froid devient alors, dans ce contexte, un enjeu scientifique et géostratégique pour les grandes puissances.

Il nous semble ici intéressant d'approfondir cette approche conflictuelle en la déplaçant dans le champ technique. Aussi peut-on se questionner sur la "guerre" technique et culturelle du froid au prisme tout d'abord de deux systèmes techniques tels que définis par Ruth Schwartz Cowan (Schwartz Cowan 1985). Les résultats des recherches de l'historienne américaine des techniques présentés dans un article intitulé «How refrigerators got its hum" (Schwartz Cowan 1985) viennent nourrir comme autant d'échos cette réflexion et inspirent l'approche développée ici. Pour cerner l'évolution de la production et de la diffusion du réfrigérateur en France, notamment à travers les stratégies de ventes développées par Manufrance, nous analyserons les oppositions entre les deux sources d'alimentation, le gaz et l'électricité, puis de deux technologies, l'absorption et la compression.

\section{Deux systèmes techniques en concurrence}

4 Au fil de ses recherches, R. Schwartz Cowan a étudié les débuts du marché du froid domestique américain et insista fortement sur la période de fracture des années 1920 . Aux États-Unis, on retrouve alors deux types de technologies appliquées à la production du froid domestique : l'absorption et la compression (fig.1 et 2). Dans les deux cas, les industriels utilisent conjointement un gaz et une source de chaleur pour produire $\mathrm{du}$ froid par liquéfaction du gaz frigorifique. Si les réfrigérateurs à compression utilisent des moteurs électriques, les réfrigérateurs à absorption, eux, utilisent principalement le gaz comme énergie, mais aussi le butane et le pétrole. 
Fig.1. Schéma du fonctionnement du réfrigérateur à absorption

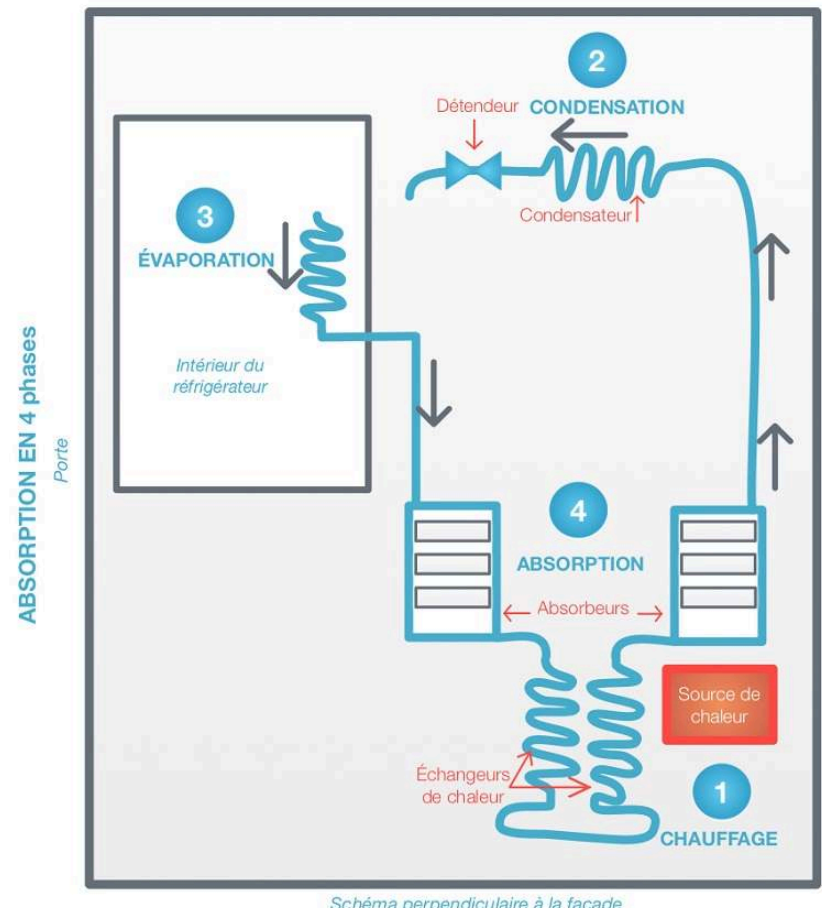

Schéma Aurélie Brayet

Fig.2. Schéma du fonctionnement du réfrigérateur à compression

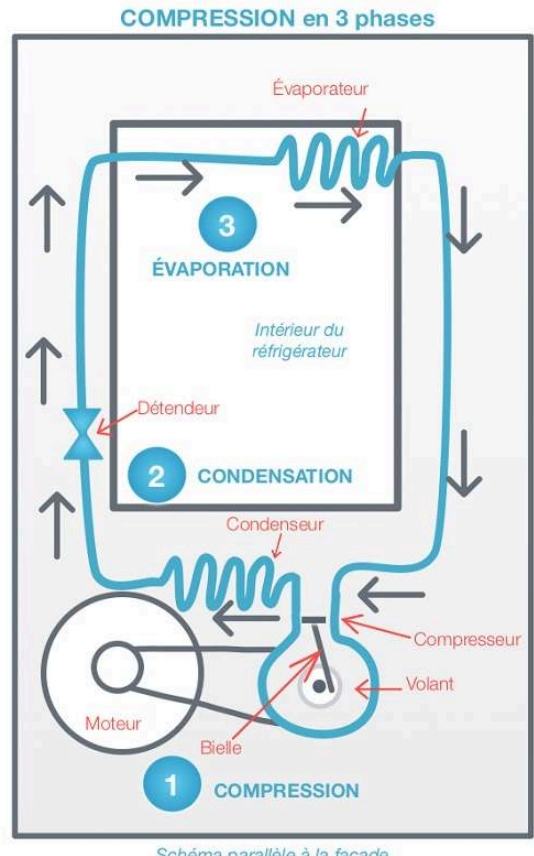

Schéma Aurélie Brayet 
5 Jusque dans les années trente, les deux technologies et les deux sources d'énergie cohabitent et se partagent le marché domestique. Par la suite, la compression et l'alimentation électrique vont dominer le marché et conduire à la disparition et de l'absorption et de l'alimentation au gaz. En s'interrogeant sur les raisons pouvant expliquer la primauté d'un modèle technique sur un autre, R. Schwartz Cowan a mis en évidence des éléments de réponse ne relevant pas de la performance technique. Techniquement viable, le système à absorption fonctionnant au gaz était même plus fiable que son concurrent dans la mesure où l'absence de parties motorisées limitait les risques de panne. R. Schwartz Cowan pose alors le concept de «Common sense machine» (Schwartz Cowan $1987: 261-280$ ) pour tenter de saisir les raisons de cette primauté. $\mathrm{Si}$, durant la période d'entre-deux-guerres, les réfrigérateurs électriques deviennent le standard américain, ce n'est donc ni pour des raisons techniques, ni pour des raisons culturelles.

6 Pour l'auteur, il s'agit davantage d'une question de pouvoir des compagnies d'électricité et notamment de General Electric (GE) qui avait développé un réseau efficace de vendeurs, revendeurs, de médiateurs pour commercialiser les produits, mais aussi pour diffuser parmi les ménages américains une culture du frigo électrique. Par ailleurs, le capital de l'entreprise était également assez important pour permettre la création d'une stratégie marketing féroce, originale et offensive ${ }^{4}:$ trains showrooms qui sillonnent le pays pour présenter le Monitor Top, présentation du millionième réfrigérateur Monitor Top à la radio par H. Ford en 1931, publicité autour du réfrigérateur offert à Robert Ripley en 1928 pour accompagner l'expédition sousmarine vers le pôle Nord. La stratégie publicitaire vise à starifier le produit en créant des événements qui marquent les esprits, et qui pour certains entreront dans l'histoire. Ainsi le réfrigérateur General Electric est la vedette du premier film publicitaire en Technicolor en 1935. L'appareil invisible de nos cuisines s'affichait alors sur grand écran dans les salles de cinéma!

7 Aussi, pour R. Schwartz Cowan, le monopole technique du réfrigérateur à compression aux États-Unis, à terme unique choix technique proposé aux Américains, est dû au conservatisme des compagnies gazières. Le refus d'investir dans l'innovation, l'absence de concurrence liée à la forte concentration de ce secteur sont les arguments majeurs énoncés. Enfin, il faut ajouter à ces causes structurelles et économiques, qui concentrent l'analyse du côté de la production, du produit et de l'entreprise, la prise en compte indispensable de l'usager-consommateur, sujet libre et agissant ${ }^{5}$.

8 Sur la base de ces premiers résultats, on peut légitimement se demander ce qu'il en a été en dehors des États-Unis? En prenant comme point de départ la forte concurrence des deux systèmes énergétiques et techniques existants en France, nous avons confronté les résultats des analyses de Cowan au marché français des années 1930 à 1980. Les sources mobilisables pour l'étude du cas français diffèrent mais sont néanmoins nombreuses. Ainsi, les stratégies publicitaires des entreprises et compagnies gazières et électriques restent en ordre dispersé, sans réelle ligne directrice $^{6}$. La lutte commerciale entre les compagnies électriques et gazières ne se focalise pas en France sur les réfrigérateurs mais plutôt sur les appareils de cuisson (four et plaque essentiellement) (Williot 2005, 2007, 2015).

9 Les archives d'EDF, par l'absence de données sur les réfrigérateurs dans les rapports, études et guides de formation des conseillères et conseillers, confirment ce désintérêt des industries gazières pour la diffusion d'appareils de conservation par le froid (Brayet 
2019 :162). Confrontés aux revues L'Art ménager (puis Arts ménagers), elles permettent de bien cerner la particularité du rapport des Français aux sources d'énergie génératrices de froid et la perception française des deux systèmes techniques: compression et absorption. Les archives Manufrance, quant à elles, se révèlent être essentielles pour appréhender à travers une lignée technique de réfrigérateurs la culture technique dominante, à savoir la réfrigération par compression.

\section{Gaz vs électricité}

Durant l'entre-deux-guerres, on retrouve dans l'hexagone la même variété de solutions techniques que celle proposée aux États-Unis et l'on peut acquérir des réfrigérateurs alimentés à l'électricité ou au gaz. À cette époque, la concurrence entre les énergies domestiques est importante (Drouard, Williot 2007 ; Beltran 2000). En juin 1927, un article publié dans la revue L'Art ménager, intitulé « Le froid artificiel chez soi, au gaz ou à l'électricité7 ", fait état de ce choix et chaque fournisseur d'énergie vante les mérites de son énergie et de son utilisation pour réfrigérer et conserver les produits alimentaires. Par exemple, en 1933, dans la revue L'Architecture, l'ingénieur en chef de la Société du gaz de Paris présente les avantages des réfrigérateurs utilisant le gaz comme source d'énergie :

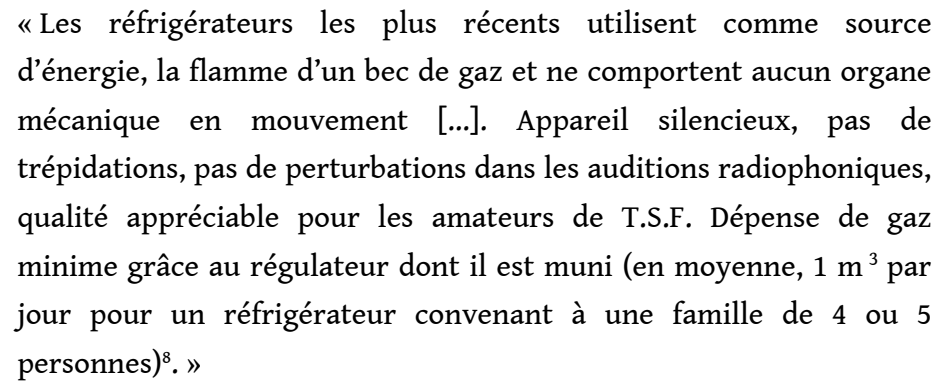

11 Ce propos est largement partagé par "L'animateur gazier », dans la revue Le gaz et l'électricité, en décembre 1934. Le journaliste insiste sur la qualité des appareils ménagers au gaz et tente de ridiculiser, non sans humour, la concurrence électrique, mettant en avant la variété des offres techniques pour le froid en France, et révélant de façon indirecte une forte dynamique pour les moteurs électriques :
«La grande presse nous parle avec les plus amples détails des "grandes conquêtes" de la radiophonie, et beaucoup moins des services réguliers du fidèle combustible fluide. [...] Autre avantage des ondes courtes : les denrées alimentaires qui seront placées dans le champ d'irradiation seront préservées de toute putréfaction. La T.S.F ne se satisfaisant pas de la musique voire de la cuisine, se mêlerait ainsi de remplacer le réfrigérateur à gaz pour la conservation des aliments. Voilà qui ne va pas mieux ${ }^{9}$.»

Si la presse des années 1930 relate les avantages et les qualités des réfrigérateurs au gaz, les statistiques EDF-GDF sur les énergies utilisées dans les foyers français entre 1950 et 1970 mettent en avant une écrasante majorité de foyers équipés de réfrigérateurs électriques ${ }^{10}$. Aussi, malgré la confiance accordée par les journalistes à l'emploi de cette énergie, les usagers eux, ne suivent pas. L'analyse des archives EDF- 
GDF et notamment celles liées aux campagnes gaz, montre que GDF, si entreprenant dans la défense de la cuisine au gaz, ne livre pas avec la même énergie la bataille pour promouvoir le froid au gaz ${ }^{11}$. Le réfrigérateur, seul produit branché en continu, trouverait pourtant un intérêt certain à être alimenté au gaz afin d'éviter les pannes et coupures d'électricité, plus fréquentes que les pannes ou coupures de gaz. Cela interroge.

De même, on peut noter que le célèbre catalogue de vente par correspondance Manufrance ne propose pas un seul réfrigérateur alimenté au gaz, alors même que sa clientèle, essentiellement rurale, constituerait une cible privilégiée pour ces appareils. Un élément de réponse peut être apporté par la lecture des enquêtes de consommation EDF-GDF. Contrairement aux usages et pratiques actuels, au moins jusqu'aux années 1970, les réfrigérateurs n'étaient que très rarement branchés en continu. En effet, les rapports EDF-GDF sur le marché du gaz et de l'électricité de l'époque insistent sur cet aspect inattendu et catégorisent le réfrigérateur dans le groupe des produits saisonniers. Ainsi, les utilisateurs en feraient davantage usage en été et ils débrancheraient volontiers l'appareil sur des périodes variables.

Dans les rapports des enquêtes menées par EDF auprès de sa clientèle en Avignon entre mai 1957 et décembre 1959, il était possible de lire : « Nous sommes en saison, le frigo ne marche pas. "; "J'ai le frigidaire, mais comme je n'ai pas le courant pilote. Il ne marche pas tellement. »; « Le frigidaire, il ne marche pas l'hiver »12. L'usage exclusif de l'énergie électrique (quel que soit le système choisi, absorption ou compression) semble au final de l'ordre du bon sens : il est plus facile et bien moins risqué de débrancher et rebrancher une prise que de couper et remettre le gaz ${ }^{13}$. Qu'en est-il, au-delà de la source d'énergie, pour les deux technologies qui se disputent le marché du froid domestique?

\section{Compression vs absorption}

15 Le monopole de la compression sur l'absorption s'impose plus tardivement sur le marché français que sur le marché américain. Au moment où, aux États-Unis, cette technologie devient hégémonique, en France, les premiers réfrigérateurs à absorption viennent seulement de faire leur apparition. Par exemple, le catalogue des exposants de réfrigérateurs au Salon des Arts ménagers entre 1923 et $1930^{14}$ indique la présence en 1927 d'un premier réfrigérateur à absorption de la marque Isber ${ }^{15}$. Puis en 1929, c'est le réfrigérateur Frigelux d'Electrolux qui est présenté.

Loin d'être majoritaires sur un marché du réfrigérateur fort limité au demeurant, les appareils à absorption apparaissent sous diverses marques relevées dans les catalogues des exposants et dans les revues Arts ménagers (publicités). En 1932, par exemple, on trouve le Frigory de la marque King of Gold (KOG), Nord-Frigor et Frigibloc de la SEV (Simon Perret), Refrigex de la Société Générale de Matériel frigorifique (Ancien Ets Hasse) et Protos Frigos. Ce dernier a l'originalité d'utiliser le courant électrique uniquement pendant quatre heures la nuit et une fois débranché, de restituer du froid pendant le reste de la journée sur le modèle de la glacière. Comme nous pouvons le voir sur le graphique (fig.2) minoritaire avant la Seconde Guerre mondiale, le nombre de ces appareils vendus dépasse celui des appareils à compression vers 1949-1950 avant de représenter, en 1950, la moitié du parc ${ }^{16}$. 
S'il est possible de lire chronologiquement l'histoire des recherches et innovations frigorifiques (Brayet 2019), l'analyse historique du marché français et du parc de réfrigérateurs en circulation échappe à toute tentative d'approche chronologique linéaire. Si des lignes de force se dégagent, les systèmes techniques abandonnés (glacière, absorption) persistent malgré l'omniprésence $\mathrm{du}$ système choisi (compression). Une immersion dans le catalogue Manufrance, aux pages " réfrigérateurs", permet d'identifier les mouvements de définition d'une culture technique.

Fig.2. Graphique des ventes annuelles de réfrigérateurs en France entre 1947 et 1957

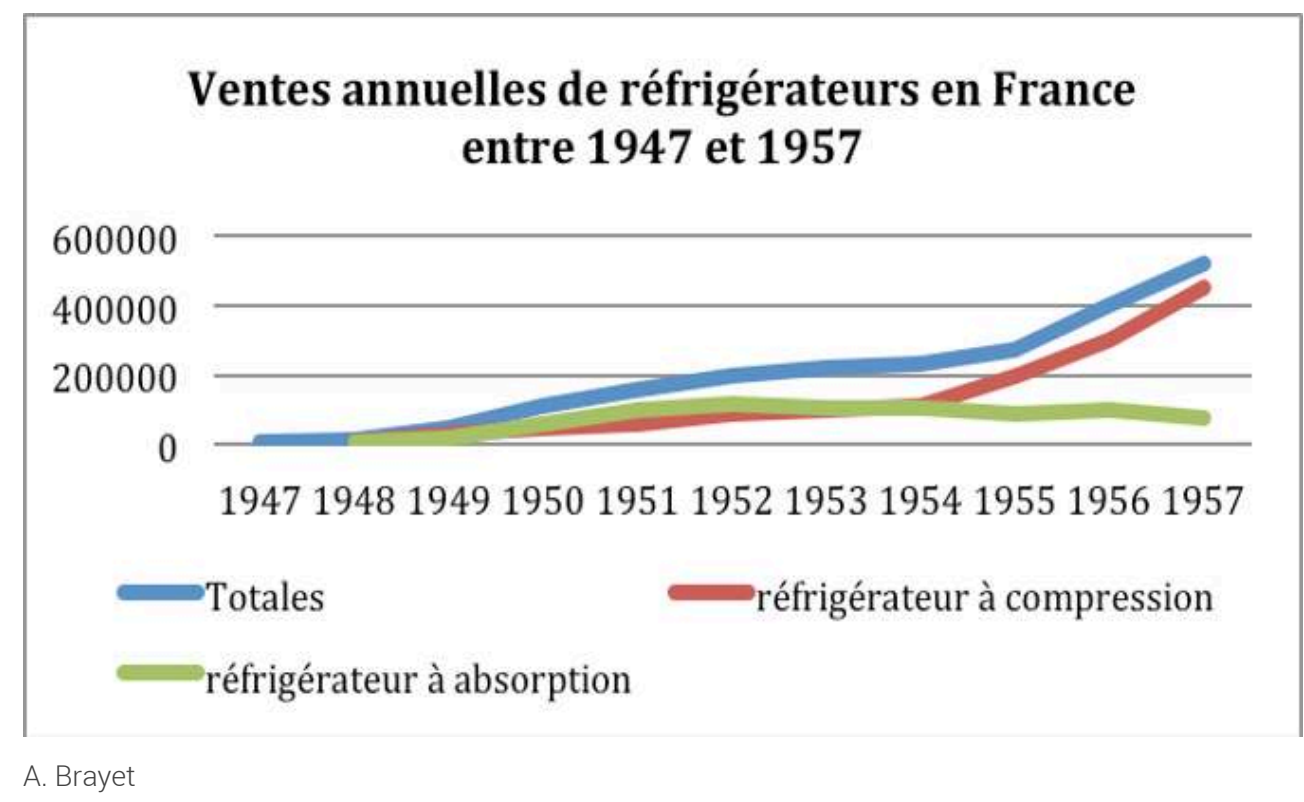

En termes de distribution, Manufrance, par exemple, dans ses catalogues, vend ses premiers réfrigérateurs dès 1936. Jusqu'en 1950, il s'agira uniquement de réfrigérateurs à compression. C'est en effet seulement en 1950 que l'entreprise stéphanoise propose un réfrigérateur à absorption. Fait étonnant, pendant trois ans, la marque ne vendra que ce système. Toutefois, à partir de 1952, elle offrira le choix à ses clients entre des réfrigérateurs à compresseurs hermétiques ou à absorption et ce jusqu'en 1962. Pourquoi ces évolutions? Rappelons tout d'abord que Manufrance distribuait sous l'estampille MF des produits fabriqués par d'autres entreprises, généralement connues pour la qualité de leurs productions ${ }^{17}$.

Grâce à ce choix, Manufrance se libère des contraintes liées à la production comme par exemple la maîtrise par un fabricant d'un seul système technique (le plus souvent en effet une marque fabrique un seul type de réfrigérateur). Manufrance peut ainsi vendre ce qui lui semble le plus en adéquation avec les besoins du marché et les attentes de ses clients $^{18}$. L'objectif est donc différent : pour Manufrance, il ne s'agit pas de convaincre les clients d'adopter un type de réfrigérateur ou un autre, de privilégier une énergie ou une technologie, mais de proposer le modèle qui plaira le plus et donc se vendra le plus ${ }^{19}$.

Entre 1936 et 1950, Manufrance s'approvisionne exclusivement en réfrigérateurs américains chez Frigidaire. Le choix de ce fournisseur s'appuie à la fois sur la réputation du fabricant et sur les attentes des consommateurs : un produit qui a fait ses 
preuves et qui inspire donc confiance aux consommateurs. Les descriptifs et arguments de vente publiés dans le catalogue vantent d'ailleurs le perfectionnement de «l'armoire frigorifique électrique ". À cette époque, les réfrigérateurs à absorption ne sont sur le marché français que depuis quatre ou cinq ans. Cette jeunesse rencontre aussi un autre aspect économique important pour l'entreprise: comme Manufrance garantit ses réfrigérateurs 5 ans, il y a alors trop peu de recul sur les garanties fournies par ce produit $^{20}$. Le choix du produit vendu est donc très important pour assurer le minimum de service après-vente et ainsi dégager de solides bénéfices.

Comme cela était précédemment esquissé, un revirement stratégique s'opère entre 1950 et 1952. Manufrance décide alors de proposer exclusivement des réfrigérateurs à absorption car ceux-ci correspondent non seulement à l'évolution du foyer, à savoir des familles moins nombreuses, avec un pouvoir d'achat moyen, mais aussi aux attentes des consommateurs en proposant un produit plus silencieux, sans entretien et dont le fonctionnement simple fait moins peur ; arguments que Manufrance développe dans la notice de présentation du réfrigérateur proposé dans son catalogue de 1950 :

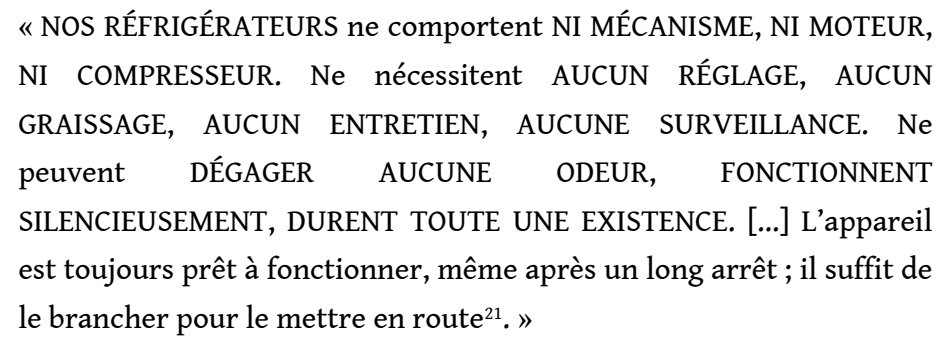

Entre 1952 et 1961, Manufrance proposait donc les deux types de réfrigérateurs avant de ne vendre en 1962 que le modèle à compresseur hermétique. Cette seconde évolution est due aux changements de contexte: entre 1949 et 1952 le nombre d'appareils vendus passe de 60000 unités à 220000 unités en France ${ }^{22}$. L'augmentation des ventes va de pair avec les attentes précises des clients en termes de capacité de stockage : à l'heure du baby-boom, les clients recherchent des réfrigérateurs de plus grandes dimensions que seul le système à compression permet alors.

L'autre argument non négligeable est que si le réfrigérateur à absorption coûte moins cher à l'achat, il utilise davantage d'électricité et revient ainsi plus cher à l'usage. Il se dégage ainsi un certain rapport des Françaises et Français à la "modernisation ", questionnant par la même la notion de "Trente Glorieuses ». La commercialisation du produit « réfrigérateur » illustre une réelle spécificité française dans la diffusion des techniques, allant à l'encontre de l'idée trop souvent admise d'une standardisation de la production des biens domestiques sur celle des États-Unis (Pessis, Topçu et Bonneuil 2016).

24 À travers cette étude hexagonale et comme R. Schwartz Cowan pour le marché étatsunien, nous parvenons à la conclusion d'une disparition des modèles à absorption ; toutefois, le faisceau d'explications diffère.

25 L'argument du manque d'innovations des producteurs de ces modèles, Servel aux ÉtatsUnis et Electrolux en France, ne peut être considéré comme une cause forte de cette désaffection. L'étude des publicités de ces deux marques et notamment des arguments de vente présentés atteste que toutes les innovations esthétiques, ergonomiques ou techniques pour accompagner des besoins annexes au stockage réfrigéré des denrées 
alimentaires (aménagement de la porte, freezer, design des poignées ou de l'habillage, fabrication de glaçons...) ont été suivies, voire anticipées.

Une première explication est à trouver dans la faiblesse de contenance et de rendement des réfrigérateurs à absorption. Par exemple, en 1957, ces derniers proposent un volume de stockage de 43 à 196 litres, contre 95 à 278 pour les modèles à compression. Considérant qu'il faut un volume de stockage d'environ 30 ou 40 litres par personne, les modèles à absorption ne répondent bientôt plus aux besoins des familles. En février 1965, L'officiel du froid et des Arts ménagers, rubrique "art-ménagisme», semble poser pour la dernière fois la question dans "L'absorption : désuétude ou résurrection? $»^{23}$. Ce questionnement s'appuyait sur les travaux de l'ingénieur suisse H. Stierling qui semblait nourrir l'espoir d'un prototype de réfrigérateur combiné et compétitif de 190 litres.

Une seconde explication peut provenir de la nature de l'utilisation du produit et des questions de sécurité liées à cette utilisation. Plusieurs articles de presse évoquent les défaillances de réfrigérateurs à absorption pour cause d'intervention par les particuliers sur le produit. En effet, le système à absorption permet aux amateurs de bricolage, qu'ils soient éclairés ou non, non seulement de «fabriquer » des appareils chez eux mais aussi de modifier ceux achetés. Bien entendu, cela conduit à quelques dysfonctionnements. Relayés par la presse et les rumeurs, ces embarras, bien que non directement liés à ce type de technique frigorifique, ne font qu'accroître leur discrédit ${ }^{24}$. C'est d'ailleurs un incident lié à la fuite de gaz d'un réfrigérateur à absorption, causant la mort d'une famille entière, qui fut à l'origine des travaux d'Albert Einstein et Léo Szilard ${ }^{25}$ sur l'invention d'un réfrigérateur totalement hermétique et sûr.

Ainsi, en France, dans un marché d'équipement ménager marqué par une lutte concurrentielle, le choix pour un plus grand volume de stockage et pour une plus grande sécurité semble sceller le sort du réfrigérateur à absorption et conduira, comme aux États-Unis, à son élimination progressive et définitive du marché domestique.

\section{BIBLIOGRAPHIE}

BELTRAN Alain, CARRÉ Patrice-Alexandre, La fée et la servante, La société française face à l'électricité, Paris, Belin, 2000

BLAIN Bodil Bjerkvik, « Melting Markets: The Rise and Decline of the Anglo-Norwegian Ice Trade, 1850- 1920 », Working Papers of the Global Economic History Network, vol. 20/06, Londres, London School of Economics, février 2006 ; URL : http://eprints.lse.ac.uk/22471/1/wp20.pdf

BRAYET Aurélie, Un Frigidaire et nous serons heureux. Histoire culturelle et technique du réfrigérateur, Belfort, Pôle éditorial de l'université de technologie de Belfort-Montbéliard, 2019

CARRIERE Bruno, « Aux origines des transports frigorifiques par rail en France », Revue d'histoire des chemins de fer, 41, 2010 ; DOI : https://doi.org/10.4000/rhcf.1179 
DROUARD Alain, WILLIOT Jean-Pierre (dir.), Histoire des innovations alimentaires, $\mathrm{XIX}^{e}-\mathrm{XX}^{e}$ siècles, Paris, L'Harmattan, 2007, p.219-242

DELAUNAY Quynh, Société industrielle et travail domestique, l'électroménager en France (XIX ${ }^{e}-X X^{e}$ siècle), Paris, L'Harmattan, 2003

EDGERTON David, Quoi de neuf? Du rôle des techniques dans l'histoire globale, Paris, Le Seuil, 2013

PESSIS Cécile, TOPÇU Sezin, BONNEUIL Christophe (dir.), Une autre histoire des « Trente Glorieuses » : modernisation, contestations et pollutions dans la France d'après-guerre, Paris, France, La Découverte, 2013

ROUAUD Jacques, 60 ans d'Arts Ménagers, Paris, Syros Alternatives, 1989, tome $1: 1923-1939$, le confort, et 1993, tome $2: 1948-1983$, la consommation, 1993

SCHWARTZ COWAN Ruth, " How refrigerators got its hum », in The Social shaping of technology, sous la direction de Donald MacKenzie et Judy Wajcman, Philadelphie, Open University Press, 1985 , p.202-218

SCHWARTZ COWAN Ruth, «The Consumption Junction: A Proposal for Research Strategies in the Sociology of Technology ", in Wiebe E. Bijker, Thomas Hugues et Trevo J. Pinch, The Social Construction of Technological Systems: New Directions in the Sociology and History of Technology, Cambridge, MIT Press, 1987, p.261-280

SCHWARTZ COWAN Ruth, More work for mother: the ironies of household technology from the open hearth to the microwave, New York, Basic Books, 1983; DOI: https://doi.org/

10.1002/1520-6696(198601)22:1<81 : :AID-JHBS2300220113>3.0.CO ;2-B

THÉVENOT Roger, Essai pour une histoire du froid artificiel dans le monde, Paris, Institut International du Froid, 1978

WILLIOT Jean-Pierre (dir.), Du feu originel aux nouvelles cuissons. Pratiques, techniques, rôles sociaux du faire cuire, Bruxelles, Peter Lang, 2015

WILLIOT Jean-Pierre, « De l'innovation industrielle à l'excellence gastronomique : la cuisine au gaz aux XIX et XX ${ }^{\mathrm{e}}$ siècles », in DROUARD Alain, WILLIOT Jean-Pierre (dir.), Histoire des innovations alimentaires, $\mathrm{XIX}^{e}-\mathrm{XX}^{e}$ siècles, Paris, L'Harmattan, 2007, p.219-242

WILLIOT Jean-Pierre, L'industrie du gaz en Europe aux XIX ${ }^{e}$ et XX $X^{e}$ siècles. L'innovation entre marchés privés et collectivités publiques, Bruxelles, Peter Lang, Euroclio, 2005

\section{NOTES}

1. Un dossier spécial sera bientôt consacré à l'étude pluridisciplinaire des arts ménagers culinaires dans la revue $e$-Phaistos : «Le goût des techniques. Arts ménagers culinaires, culture et technique ». Nous reviendrons dans ce dossier sur l'historiographie de cette thématique.

2. Le développement de la fabrication et diffusion de nouveaux produits agroalimentaires (avec notamment les crèmes glacées), le développement de la pêche, l'essor des brasseries et la demande croissante en bière blonde (la fermentation de la bière blonde se produit à 5-8 degrés contrairement aux bières anglaises pour lesquelles la fermentation est à 20-30 $)$ et la structuration et déploiement de la filière viande sont des industries qui favorisent l'augmentation du besoin en froid.

3. Roger Thévenot parle d'une "guerre" du froid pour évoquer la conquête de nouveaux marchés lointains grâce au transport maritime frigorifique. Nous parlons ici de «guerres » au 
pluriel pour rendre compte des luttes acharnées pour le monopole technique d'un seul système de froid électrique à compression.

4. Elle parle d' " outlandish advertising and public relations techniques ».

5. R. Schwartz Cowan développera en 1987 le concept de « consumption junction » et plaidera pour la prise en compte du consommateur dans l'histoire des techniques.

6. Nous conduisons actuellement une étude sur la représentation du réfrigérateur dans la bande dessinée. Si la constitution du corpus de planches de bandes dessinées souligne également l'absence d'une stratégie globale, la richesse et la variété de celui-ci montre l'intérêt, la curiosité et les espoirs que soulèvent cet objet qui semble fasciner les auteurs et dessinateurs. DanièleAlexandre Bidon, spécialiste de l'histoire de la cuisine médiévale et de la bande dessinée, a attiré notre attention sur cet axe de recherche et nous accompagne dans l'étude des planches.

7. Archives de l'Association Arts Ménagers (AAM). Revue L'Art ménager. «Le froid artificiel chez soi, au gaz ou à l'électricité », par Charles Pierson, juin 1927.

8. «La cuisine et les appareils » par Reclus, ingénieur en chef de la Société du gaz de Paris, in L'Architecture, revue mensuelle, Supplément technique n¹4, décembre 1933-janvier 1934.

9. Collection personnelle. "L'animateur gazier », revue Le gaz et l'électricité, déc. 1934.

10. Archives EDF : 800998 : documents publicitaires du SAM 58; 823034 : Campagne et Centre 1956-1957; 823499: Le marché domestique du gaz et de l'électricité et la concurrence, comparaison internationale, 1970.

11. Archives EDF : 800994 : Protocole national 1951 ; 823034 : Campagne gaz 1956 rapport annuel ; 823503 : Campagne gaz 1972 rapport annuel ; 800741 : Analyse de l'équipement ménager, 1959 ; 823035 : Annonces et éléments publicitaires des campagnes de propagande, 1949-1957, BIP.

12. Archives EDF : 823499: "Les effets de l'action commerciale sur le marché domestique de l'électricité", étude de UNIMAL (Union pour l'étude du marché de l'électricité), Avignon, 1957-1959, p.29.

13. Archives EDF : 823499 : Le marché domestique du gaz et de l'électricité et la concurrence, comparaison internationale, 1970. Rapport de la Direction de la distribution du 9 juin 1970.

14. Archives de l'AAM, Catalogue du Salon des Arts ménagers.

15. On trouve d'ailleurs une publicité pour le réfrigérateur à absorption dans L'Art ménager d'août 1927. Archives de l'AAM. Revue L'Art ménager.

16. Archives du Musée de la publicité, Paris, «Réfrigérateur », revue Vendre, juin 1957, n 328, p. 45. Un rapport intitulé «Analyse de l'équipement ménager selon les CSP, 1959 » dans les Archives EDF (800998) montre que 1,369 millions de réfrigérateurs électriques, représentant 96\% du parc et comportent : $47 \%$ d'appareils à absorption (87\% de moins de 1001$)$ et $53 \%$ d'appareils à compression (82\% au moins égale à 1001).

17. Archives départementales de la Loire (ADL), Fonds Manufrance : 63J1 : Statut de l'entreprise.

18. ADL, Fonds Manufrance.

19. L'étude de 1958 sur le marché du réfrigérateur menée par EDF montre que les foyers ruraux possèdent plus d'appareils à compression. Ceci est dû à la proportion élevée d'appareils de grandes capacités en milieu rural. Archives EDF, Données récentes sur le marché du réfrigérateur, 1958, 24p.

20. ADL 63J7549, Réfrigérateurs.

21. Fonds ancien, Médiathèque Tarentaize, Saint-Étienne, Catalogue de la Manufacture d'Armes et de Cycles de Saint-Étienne, 1950, p.331.

22. Archives AAM, Dossier « froid ».

23. Archives Association Arts ménagers. Dossier «froid». L'officiel du froid et des Arts ménagers, rubrique « art-ménagisme ", février 1965, p.5-9.

24. Voir Archives du Musée de la publicité de Paris, «Réfrigérateur », revue Vendre, juin 1957, $\mathrm{n}^{\circ} 328, \mathrm{p} .49$. 
25. Pour étudier l'apport d'Albert Einstein et Léo Szilard, les sources suivantes ont été utilisées : Les dossiers des deux brevets déposés par Léo Szilard et Albert Einstein, Rapports techniques + photographie des prototypes dans les archives de l'ingénieur Korodi, Budapest (Bibliothèque de Budapest), Correspondance de Szilard, Université de San Diego: http://library.ucsd.edu/ speccoll/findingaids/mss0032.html\#header, Archives Einstein, Université hébraïque de Jérusalem : http://einsteinpapers.press.princeton.edu/ http://www.alberteinstein.info/vufind1/ Search/Results?lookfor=refrigerator\&type=AllFields\&subm it $=$ Find.

\section{RÉSUMÉS}

Négligés par l'histoire des techniques, les objets de la vie domestique et du quotidien sont pourtant des objets techniques omniprésents dans la maison et particulièrement en cuisine. Le réfrigérateur est un de ces objets invisibles. Cet humble « frigo », que nous ne voyons plus, objet d'attentions des constructeurs et de désir pour des millions d'hommes et de femmes dans les années 1950, est un objet technique, culturel et social complexe. Deux systèmes techniques (absorption et compression) ont été au cœur d'une véritable guerre du froid. Initiée par une étude américaine menée par Ruth Schwartz Cowan, l'analyse de ce conflit des techniques est ici menée sur le terrain français pour comprendre les raisons de l'hégémonie technique des réfrigérateurs à compression en France aujourd'hui.

Neglected by the history of technology, domestic and everyday objects are nevertheless omnipresent technical objects in our homes and particularly in the kitchen. The refrigerator is one of these invisible objects. This humble "fridge", which we no longer see, the object of manufacturers' attention and desire for millions of men and women in the 1950s, is a complex technical, cultural and social object. Two technical systems (absorption and compression) have been at the heart of a real cold war. Initiated by an American study led by Ruth Schwartz Cowan, the analysis of this conflict of techniques is here carried out on the French terrain to understand the reasons for the technical hegemony of compression refrigerators in France today.

\section{INDEX}

Mots-clés : histoire des techniques, arts ménagers, réfrigérateur, compression, cuisine, culture matérielle, usage

Thèmes: Un objet une technique

Keywords : history of technology, domestic arts, refrigerator, compression, kitchen, material culture, use

\section{AUTEUR}

\section{AURÉLIE BRAYET}

Docteure en histoire des techniques et titulaire d'un Master professionnel en gestion et valorisation des patrimoines, Aurélie Brayet s'intéresse à l'histoire des arts ménagers et plus spécifiquement aux objets culinaires. Chercheure associée au sein de RECITS-FEMTO-St 
(UMR6174), elle est l'auteure de plusieurs ouvrages sur l'histoire et le patrimoine des arts ménagers. 